\title{
Evaluation of the Accuracy of the Fluorodeoxyglucose-Positron Emission Tomography ([18F] FDG-PET CT) in the Diagnosis of Chronic Osteomyelitis
}

\author{
Ismael M. Sharaf, Mohamed R. Hassan, Ahmed A. Elsheikh
}

Department of Orthopedic Surgery, Faculty of Medicine, Benha University, Egypt.

Correspondence to: Ismael M. Sharaf, Department of Orthopedic Surgery, Faculty of Medicine, Benha University, Egypt.

\section{Email:}

ismaelsharaf0@gmail.com

Received: 3 October 2020

Accepted: 7 August 2021

\begin{abstract}
Chronic osteomyelitis is an infection of bone and bone marrow. Diagnosis is based mainly on the correlation between history, clinical picture, lab. analysis, bacteriological, pathological, and imaging studies. Bone biopsy for culture and sensitivity is the gold standard for the correct identification of the causative organism. The present retrospective study aims to evaluate the accuracy of FDG PET-CT in the diagnosis of COM in 18 patients who underwent FDG-PET/CT scanning for clinically or radiologically suspected COM of the lower extremity. Fourteen patients had septic non-union, three patients with aseptic non-union, and one with chronic diffuse sclerosing $\mathrm{OM}$ of Garre. Seven patients had implants at site of examination at the time of the scan. Diagnosis of COM was confirmed by deep surgical cultures and pathological analysis following PET/CT scanning. FDG-PET uptake was measured by SUV max, and we compared SUV max at the infected site and the contralateral side (SUV ratio). The sensitivity, specificity, accuracy, PPV and NPV of PET SUV max were 100\%,
\end{abstract} $66.7 \%, 94.44 \%, 93.75 \%$ and $100 \%$ respectively in the diagnosis of COM at a cut-off value of 4.46. The present study included 15 true positive, two true negative and one false-positive PET/CT results. 18F-FDG PET/CT is a highly sensitive and specific method for the evaluation of chronic osteomyelitis in patients with or without trauma. PET/CT provides anatomical localization and characterization of the infected area and has a crucial role in preoperative planning with a high degree of accuracy.

Keywords: Fluorodeoxyglucose; Positron Emission Tomography; PET CT; Osteomyelitis 


\section{Introduction}

Osteomyelitis is an infection of bone and bone marrow that can be limited to a single portion of the bone or can involve several regions, such as marrow, cortex, periosteum, and surrounding soft tissue (1). The root words osteon (bone) and myelo (marrow) are combined with itis (inflammation) to define the clinical state in which bone is infected with microorganisms (2). Furthermore, it is defined as acute or chronic inflammation of the bone, localized or generalized due to infection, usually by pyogenic organisms $(3,4,5)$.

Diagnosis of osteomyelitis remains challenging due to the absence of clear clinical, radiological, and laboratory findings in many cases (6). Although many techniques have been proposed for the noninvasive evaluation of chronic osteomyelitis, clinicians are still challenged with an indeterminate diagnosis in many cases, especially in early infections.

Preoperative planning in patients with suspected chronic osteomyelitis is complicated and the definitive required surgical procedure can often only be identified based on intraoperative findings. So, the need for a noninvasive technique having high accuracy in diagnosis and preoperative planning has become clinically essential. Defining a noninvasive diagnostic technique is paramount for establishing an accurate preoperative plan.

It has been reported that FDG-PET/CT is an excellent diagnostic tool for suspected implant-associated infections after trauma surgery and musculoskeletal infections without prior surgery $(7,8,9)$. It is not only useful for the detection of acute infections, but also it was suggested to be the most accurate imaging modality for confirming or excluding the diagnosis of low-grade infection and chronic osteomyelitis (8).

The present retrospective study aims to evaluate the accuracy of FDG PET-CT in the diagnosis of COM.

\section{Patients and methods}

This retrospective study was done on 18 consecutive patients who underwent FDG-PET/CT scanning for clinically or radiologically suspected $\mathrm{COM}$ of the lower extremity. This study was approved by the Research Ethics Committee (REC) Faculty of Medicine, Benha University with code No. MS 441-2020. There were fourteen patients who had septic non-union, one with 
chronic diffuse sclerosing OM of Garre, and three patients with aseptic nonunion.

Suspicion of COM was based on clinical signs and plain X-ray. 13 patients had positive clinically inflamed skin and draining sinus tract, one patient with inflamed skin while pus drainage stopped shortly before the scan, another patient with skin defect about $2 * 2 \mathrm{~cm}$ at the infected site and three patients had no clinical signs of infection.

All patients had positive radiological signs of infection like periosteal elevation, involucrum, and sequestrum. Definite diagnosis of COM was made based on deep surgical cultures and pathological analysis following the surgical debridement.

\section{Inclusion criteria:}

Patients included in this study were highly suspected for COM of the appendicular skeleton by clinical examination and plain X-ray. Any age group and both genders are included. We included also patients with or without metalwork in the bone.

\section{Exclusion criteria:}

Patients with clinically suspected COM of the lower extremity were excluded from the study if they had diabetic foot, prosthetic loosening, spine infections or refused to take part in the study.

Informed consent was obtained from all subjects included in the study.

All patients included in the study were evaluated and treated as regards:

1. Clinical evaluation: Detailed history and clinical examinations

2. Laboratory investigations, including complete blood picture (CBC), erythrocyte sedimentation rate (ESR), C-reactive protein (CRP) which reflect different degrees of inflammation.

3. Plain radiography

4. PET-CT scan

5. Surgical debridement

6. Microbiological examination (five deep samples form the suspected areas according to the PET CT)

7.Histopathological examination (one deep sample)

\section{Statistical analysis:}

Data were fed to the computer and analyzed using IBM SPSS software package version 20.0. (Armonk, NY: IBM Corporation). Comparisons of variables between clinically infected and normal contralateral sides were 
performed using the t-test. Quantitative data were described using range (minimum and maximum), mean, standard deviation and median. Qualitative data were described using the number and percent. The Shapiro-Wilk test was used to verify the normality of distribution for non-parametric ones. The accepted level of significance in this work was stated at 0.05 (Probability value $[\mathrm{P}$-value] $<0.05$ was considered significant). Sensitivity, specificity, accuracy, positive predictive value (PPV), and negative predictive value (NPV) were calculated by comparing the PET results to pathology.

\section{Results}

The present study included 18 patients. They were 16 males and two females with age group ranged between 7-73 years $($ mean $=40.83 \pm 17.29$ years $)$. Fourteen patients had septic non-union, one with chronic diffuse sclerosing $\mathrm{OM}$ of Garre, and three patients with aseptic non-union.

In this study, the tibia was the most common bone affected in nine patients; femur was affected in seven patients, while fibula and subtalar joint were affected in one patient.
Our study included different types of COM (according to Cierny classification) (4). We included eight patients with diffuse, five with localized and two with intramedullary COM. The most common cause of $\mathrm{OM}$ among patients was postoperative after surgical fixation of closed fractures (12 patients), while two patients due to history of open fracture and one patient had chronic diffuse sclerosing OM of Garre.

Infection was clinically evident between 2 and 60 weeks $($ mean $=15.81 \pm 17.69$ weeks) after the date of injury. Duration of infection (from date of the first infection till the index debridement) ranged from 7 to 134 months (mean = $31.36 \pm 34.5$ months). With previous operations ranging from 0-7 times (mean $=2.33 \pm 1.78$ ) before index debridement .

The PET-CT scan findings in patients with $\mathrm{COM}$ are presented in tables 1 and 2. The mean SUV max on the affected side was $9.55 \pm 5.22$. While mean SUV max on the contralateral healthy side was $1.82 \pm 0.98$. SUV max ratio between both sides was $5.93 \pm 3.33$. The pattern of FDG-uptake was diffuse in nine (50\%), localized in seven $(38.9 \%)$, and intramedullary in two (11.1\%) patients. DG-uptake length was $2.85 \pm 1.83$. 
Statistical comparisons between groups are shown in table 3 . SUV max on the affected side was significantly higher By plotting ROC curve, the sensitivity, specificity, accuracy, PPV and NPV of SUV max on affected side were $100 \%$, $66.7 \%, 94,44 \%, 93.75 \%$ and $100 \%$ than SUV max on the contralateral healthy side $\left(\mathrm{t}=7.083^{*}, \mathrm{p}<0.001 * 95 \%\right.$ CI $5.427-10.032)$.

respectively in discriminating patients with COM from patients without $\mathrm{COM}$ at a cut-off value of 4.46 (AUC $=0.87$, 95\% CI, 0.63-1.00, $\mathrm{P}=0.05$ ) (Figure 1).

Table (1) Descriptive analysis of the studied cases according to PET-CT SUV max

\begin{tabular}{llll}
\hline PET-CT-SUV max & Range & Mean & Median (IQR) \\
Affected side & $2.1-21.14$ & $9.55 \pm 5.22$ & $8.11(5.76-12.72)$ \\
$\begin{array}{l}\text { Contralateral side } \\
\begin{array}{l}\text { Ratio bet. both sides(SUV } \\
\text { ratio) }\end{array}\end{array}$ & $0.55-3.83$ & $1.82 \pm 0.98$ & $1.49(0.96-2.52)$ \\
\hline
\end{tabular}

Table (2) Distribution of the studied cases according to the description of PET-CT findings

\begin{tabular}{llll}
\hline Description & No. & \% \\
\hline Pattern of uptake & Diffuse & 9 & $50 \%$ \\
& Localized & 7 & $38.9 \%$ \\
& Intramedullary & 2 & $11.1 \%$ \\
& Total & 18 & $100 \%$ \\
FDG-uptake length $(\mathbf{c m})$ & Range & & $1-7.5$ \\
& Mean & & $2.85 \pm 1.83$ \\
& Median (IQR) & & $2.25(1.06-4.32)$ \\
\hline
\end{tabular}

Table (3) Comparison between PET-CT-SUV max on the affected side and contralateral side

\begin{tabular}{|c|c|c|c|c|c|}
\hline SUV max & Affected side & Contralateral side & $\mathrm{T}$ & $P$ & $\begin{array}{l}95 \% \text { Confidence } \\
\text { Interval of the } \\
\text { Difference }\end{array}$ \\
\hline Range & $2.1-21.14$ & $0.55-3.83$ & & & \\
\hline Mean & $9.55 \pm 5.22$ & $1.82 \pm 0.98$ & $7.083^{*}$ & $<0.001^{*}$ & $5.427-10.032$ \\
\hline Median & 8.11 & 1.49 & & & \\
\hline
\end{tabular}




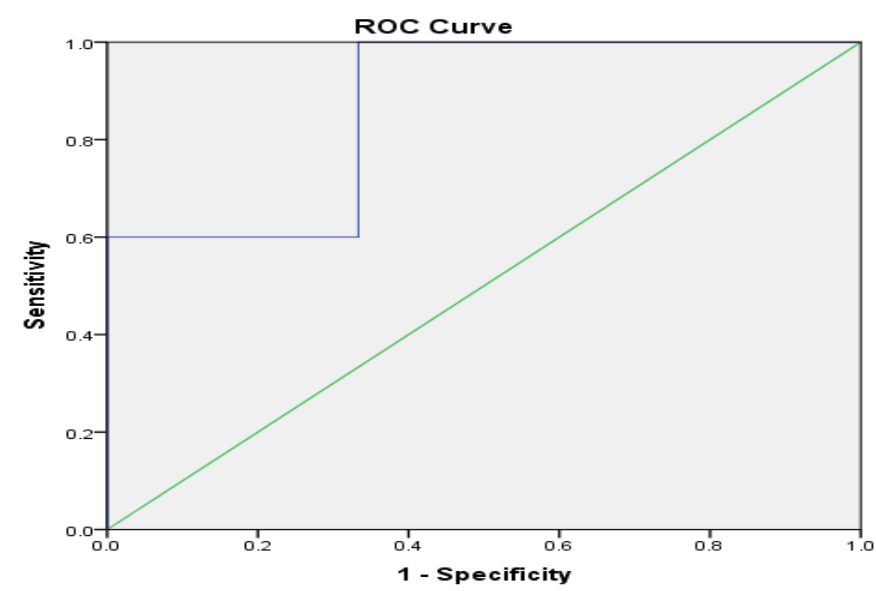

Fig 1 Receiver operating characteristic (ROC) curve shows that PET-CT-SUV max on the affected side can be used in discriminating patients with chronic OM from patients without chronic OM;

\section{Discussion}

In this study, PET-CT proved to be a highly sensitive tool in detecting COM. Additionally, it presents a three dimensional tool to understand and evaluate the distribution of infection. It also differentiates specially between soft tissue and bone infections.

Guhlmann.et al (10) investigated 31 patients suspected to have $\mathrm{COM}$ in the peripheral $(\mathrm{n}=21)$ or central $(\mathrm{n}=$ 10) skeleton and were evaluated prospectively with FDG PET. The results showed that overall sensitivity, specificity, and accuracy of PET scan were $100 \%, 92 \%$, and $97 \%$, respectively. The only false-positive finding was a patient with a soft tissue infection which on PET scan was assigned to the bone because of missing anatomical landmarks. With the use of PET-CT in the present study, the additional information provided by $\mathrm{CT}$ enabled accurate differentiation between osteomyelitis and soft tissue infection. The present study avoided this weak point which was clearly illustrated in patient No.15, whose PET-CT showed SUV $\max$ at the infected area was 6.13 in bone and 14.96 in the soft tissue around. Thus PET-CT may be used to differentiate between bone and soft tissue infections.

The CT component provided complementary information relevant to surgical planning, which minimized surgical intervention. Furthermore, PET/CT enabled the surgeon to determine the precise 
extent of resection which is illustrated by the distribution area and length of FDG uptake and helped the anticipation of the appropriate surgical approach.

Guhlmann et al. (10) used SUV mean analysis of FDG uptake for diagnosing osteomyelitis explaining the resulted very high sensitivity, specificity, and diagnostic accuracy. Their mean SUV in COM zones was $3.6 \pm 2.0$. In the current study, the analysis was based on SUV max. The mean SUV max in our study was 9.55 \pm 5.22 , which is not comparable to Guhlmann et al results.

Van Vliet et al. (11) retrospectively studied 135 patients suspected of fracture-related infections. Qualitative assessment of 18F-FDG PET/CT scans showed a sensitivity of $89 \%$, specificity of $80 \%$, PPV of $74 \%$, NPV of $91 \%$ and diagnostic accuracy of $83 \%$.

A meta-analysis done by Wang et. al. (12) included seven articles.18F-FDG PET/CT scans for 319 patients showed $92.3 \%$ sensitivity and 92\% specificity in COM diagnosis.

Schiesser et al studied FDG PET scans in 22 patients suspected of having metallic implant-associated infections 14 were true-positive, 14 were true-negative, and one was false-positive. Sensitivity, specificity, and accuracy were $100 \%, 93.3 \%$, and 97\%, respectively, for all PET data; $100 \%, 100 \%$, and $100 \%$, respectively, for the central skeleton; and 100\%, $87.5 \%$, and $95 \%$, respectively, for the peripheral skeleton (9). The degree of overall interobserver concordance was high, and their study included soft tissue infections as well, which could explain the higher diagnostic accuracy.

Unlike MRI and CT, FDG PET images do not show implantassociated artefacts that can interfere with image interpretation in trauma patients with osteosynthetic metallic implants. Schiesser et al. (9) emphasized that FDG PET was not affected by artefacts from metallic implants used for fixation of fractures, which is concordant with previously reported studies $(10,13)$. This is consistent with the present study, which included seven patients with implants in the area of interest at the time of PET scan performance.

It is well known that postoperative reparative tissue and fractures may present with increased FDG uptake $(14,15)$. Increased FDG uptake 
normalizes nearly four months after traumatic or surgical fractures (16). The healing process demonstrates most of the cellular components that are present in inflammation (17).

Our data confirms this result as patient No.4 had RTA in May 2017, resulting in bilateral fracture calcaneus. The right calcaneus had an open fracture, and it was treated with no complications while the left calcaneal fracture was closed and treated conservatively resulting in malunion and subtalar arthritis. Then arthrodesis was applied and complicated by COM. SUV max in the right calcaneus was $2.61(55.29 \%$ of the infected left subtalar joint 4.72).

Hartmann et al, (18) studied 33 patients with trauma suspected of having chronic osteomyelitis. The infection was suspected in the axial and appendicular skeleton in 10 and 23 patients, respectively. PET/CT was performed in 18 patients in the presence of metallic implants. Histopathology or bacteriological culture was used as the standard of reference.

They found 17 were true positive, 13 true negative, two false positives and one false negative. 18 patients had chronic osteomyelitis and 15 had no osseous infection according to the reference standard. Sensitivity, specificity and accuracy for (18)FFDG PET/CT was 94\%, 87\% and $91 \%$ for the whole group, $88 \%, 100 \%$ and $90 \%$ for the axial skeleton and $100 \%, 85 \%$ and $91 \%$ for the appendicular skeleton, respectively (18).

The one false-positive finding was due to a non-union fracture of the femur with negative microbiological results. The CT component of the scan yielded the diagnosis of a nonunion, demonstrating prominent cortical bone within the marrow cavity (18). These data were consistent with the present study.

Hartmann et al (18) used visual analysis for FDG uptake. Wenter et al used visual assessment in addition to SUV measurements and found sensitivity, specificity, and diagnostic accuracy of 85,86 , and $86 \%$ respectively (19). The visual scoring of FDG-uptake is less objective than the use of SUV max and may be more sensitive to inter- and even intraobserver differences (20). In our study, we performed quantitative SUV max based analysis. 
Wenter et al studied thirty patients retrospectively described as, 13 aseptic delayed unions and 17 septic delayed unions. Sensitivity, specificity and diagnostic accuracy of FDG-PET/CT were $65 \%, 77 \%$ and $70 \%$, respectively with a cut-off SUV $\max$ set at $4.0(19)$.

In a study conducted by De Wenter et al (7) the overall sensitivity, specificity, and accuracy of FDG PET in detecting suspected chronic musculoskeletal infection of the appendicular and axial skeleton were $100 \%, 88 \%$, and $93 \%$, respectively.

The different diagnostic accuracy of FDG-PET/CT for detecting osteomyelitis of the present study in comparison to previous studies may be explained by differences in the patient population, sample size, inclusion criteria, and the different methods used to quantify FDGuptake. Our results had a sensitivity of $100 \%$, while the reported sensitivity ranged from $65 \%-100 \%$, specificity of $66.7 \%$ which is a little pet lower than the reported specificity which ranged from $77 \%-92 \%$, and an accuracy of $94.44 \%$ while the reported accuracy ranged from $70 \%$ $97 \%$.
In a retrospective study done by Demirev et al., 26 patients were included. Both MRI and [18 F] FDG ппт/CT were shown to be accurate in 105 qualitative detection of COM. Using the SUV max at a cut-off value of three gave optimal results (a specificity of $90 \%$ with a sensitivity of $88 \%$ ), while the use of SUV max ratio gave a worse performance. This study also revealed that the two simulated imaging strategies (MRI and FDG PET/CT) showed no difference in the final diagnosis (20).

\section{Limitations}

It is a retrospective study with all its known sources of prejudice and the small number of patients. To recommend the use of FDG-PET/CT as a diagnostic tool of $\mathrm{COM}$, larger prospective studies should be performed.

\section{Conclusion}

18F-FDG PET/CT is a highly sensitive and specific method for the evaluation of chronic osteomyelitis in patients with or without trauma. PET/CT provides anatomical localisation and characterization of the infected area and has a very important role in preoperative 
planning with a high degree of accuracy.

\section{References}

1. Lew PDP, Waldvogel PFA. Osteomyelitis. Lancet. 2004;364(9431):369-79.

2. Zuluaga AF, Galvis W, Jaimes F, Vesga O. Lack of microbiological concordance between bone and non-bone specimens in chronic osteomyelitis: An observational study. BMC Infect Dis. 2002;2.

3. Jacobson A. Dorland's Pocket Medical Dictionary, 26th edition. Am J Orthod Dentofac Orthop. 2001;120(4):454.

4. Waldvogel FA, Medoff G, Swartz MN. Osteomyelitis: A Review of Clinical Features, Therapeutic Considerations and Unusual Aspects. N Engl J Med. 1970;282(4):198-206.

5. CIERNY, III G. A clinical staging system for adult osteomyelitis. Contemp Orthop. 1985;10:17-37.

6. Zhuang H, Duarte PS, Pourdehand M, Shnier D, Alavi A.: Exclusion of Chronic Osteomyelitis With F-18 Fluorodeoxygluc... : Clinical Nuclear Medicine. Clin Nucl Med J. 2000;25(4):281-4.

7. De Winter F, Van De Wiele C, Vogelaers D, De Smet K, Verdonk R, Dierckx RA. Fluorine-18 fluorodeoxyglucose-positron emission tomography: A highly accurate imaging modality for the diagnosis of chronic musculoskeletal infections. J Bone Jt Surg Ser A. 2001;83(5):651-660+ADV 24.
8. Termaat MF. The Accuracy of Diagnostic Imaging for the Assessment of Chronic Osteomyelitis: A Systematic Review and Meta-Analysis. J Bone Jt Surg. 2005;87(11):2464.

ser M, Stumpe KDM, Trentz O, ann T, Von Schulthess GK. Detection ve unuallic implant-associated infections with FDG PET in patients with trauma: Correlation with microbiologic results. Radiology. 2003;226(2):391-8.

10. Guhlmann A, Brecht-Krauss D, Suger G, Glatting G, Kotzerke J, Kinzl L, et al. Chronic osteomyelitis: Detection with FDG PET and correlation with histopathologic findings. Radiology. 1998;206(3):749-54.

11. JVC L, MGG H, FFA Ij, JDJ P, J van den $\mathrm{K}, \mathrm{P} \mathrm{B}$, et al. The diagnostic accuracy of 18 F-FDG PET/CT in diagnosing fracturerelated infections. Eur J Nucl Med Mol Imaging. 2019;46(4).

12. Wang G, Zhao K, Liu Z, Dong M, Yang S. A meta-analysis of fluorodeoxyglucosepositron emission tomography versus scintigraphy in the evaluation of suspected osteomyelitis. Nucl Med Commun. 2011;32(12):1134-42.

13. Kälicke T, Schmitz A, Risse JH, Arens S, Keller E, Hansis M, et al. Fluorine-18 fluorodeoxyglucose PET in infectious bone diseases: Results of histologically confirmed cases. Eur J Nucl Med. 2000;27(5):524-8.

14. Meyer M, Gast T, Raja S, Hubner K. Increased F-18 FDG accumulation in an acute fracture. Clin Nucl Med. 1994;19(1):13-4. 
15. Fayad LM, Kawamoto S, Kamel IR, Bluemke DA, Eng J, Frassica FJ, et al. Distinction of long bone stress fractures from pathologic fractures on cross-sectional imaging: How successful are we? Vol. 185, American Journal of Roentgenology. 2005. p. $915-24$.

16. Kaim AH, Gross T, von Schulthess GK. Imaging of chronic posttraumatic osteomyelitis. Eur Radiol. 2002;12(5):1193202.

17. Henry G, Garner WL. Inflammatory mediators in wound healing. Surg Clin North Am. 2003;83(3):483-507.

18. Hartmann A, Eid K, Dora C, Trentz O, Von Schulthess GK, Stumpe KDM.
Diagnostic value of 18F-FDG PET/CT in trauma patients with suspected chronic osteomyelitis. Eur J Nucl Med Mol Imaging. 2007;34(5):704-14.

19. Wenter V, Albert NL, Brendel M, Fendler WP, Cyran CC, Bartenstein $\mathrm{P}$, et al. $[18 F]$ FDG PET accurately differentiates infected and non-infected non-unions after fracture fixation. Eur J Nucl Med Mol Imaging. 2017;44(3):432-40.

20. Demirev A, Weijers R, Geurts J, Mottaghy F, Walenkamp G, Brans B. Comparison of [18 F]FDG PET/CT and MRI in the diagnosis of active osteomyelitis. Skeletal Radiol. 2014;43(5):665-72.

To cite this article: Mohamed R. Hassan, Ahmed A. Elsheikh, Ismael M. Sharaf. Evaluation of the Accuracy of the Fluorodeoxyglucose-Positron Emission Tomography ([18F] FDG-PET CT) in the Diagnosis of Chronic Osteomyelitis.: A Prospective Study. BMFJ 2021: 38(orthopedic surgery): 97-107, DOI: 10.21608/bmfj.2021.44908.1321 
Benha medical journal vol. 38, special issue (orthopedic surgery), 2021 